\title{
Integrated Docking Simulation and Testing with the Johnson Space Center Six-Degree-of-Freedom Dynamic Test System
}

\author{
Jennifer D. Mitchell ${ }^{1}$, Scott P. Cryan ${ }^{1}$, Kenneth Baker ${ }^{1}$, Toby Martin ${ }^{1}$, Robert Goode ${ }^{1}$, \\ Kevin W. Key ${ }^{2}$, Thomas Manning ${ }^{2}$, and Chiun-Hong Chien ${ }^{3}$ \\ ${ }^{1}$ NASA Johnson Space Center, Houston, TX 77058 \\ ${ }^{2}$ L-3 Communication L3 Communications - Titan Group, 1002 Gemini Avenue, Suite 200, Houston, Texas 77058 \\ ${ }^{3}$ GeoControl Systems Inc., 2900 Woodridge Dr. Suite 100, Houston, TX 77087
}

\begin{abstract}
The Exploration Systems Architecture defines missions that require rendezvous, proximity operations, and docking (RPOD) of two spacecraft both in Low Earth Orbit (LEO) and in Low Lunar Orbit (LLO). Uncrewed spacecraft must perform automated and/or autonomous rendezvous, proximity operations and docking operations (commonly known as Automated Rendezvous and Docking, AR\&D). The crewed versions may also perform AR\&D, possibly with a different level of automation and/or autonomy, and must also provide the crew with relative navigation information for manual piloting. The capabilities of the RPOD sensors are critical to the success of the Constellation Program; this is carried as one of the CEV Project top risks. The Exploration Technology Development Program (ETDP) AR\&D Sensor Technology Project seeks to reduce this risk by increasing technology maturation of selected relative navigation sensor technologies through testing and simulation. One of the project activities is a series of "pathfinder" testing and simulation activities to integrate relative navigation sensors with the Johnson Space Center Six-Degree-of-Freedom Test System (SDTS). The SDTS will be the primary testing location for the Orion spacecraft's Low Impact Docking System (LIDS). Project team members have integrated the Orion simulation with the SDTS computer system so that real-time closed loop testing can be performed with relative navigation sensors and the docking system in the loop during docking and undocking scenarios. Two relative navigation sensors are being used as part of a "pathfinder" activity in order to pave the way for future testing with the actual Orion sensors. This paper describes the test configuration and test results.
\end{abstract}

Keywords: Navigation, testing, relative navigation sensors, hardware-in-the-loop, simulation, rendezvous, docking. PACS: 07.07.Df, 07.05.Hd, 07.05.Kf , 07.05.Tp, 07.87.+v.

\section{INTRODUCTION}

AR\&D for the CEV requires a suite of relative navigation sensors that meet performance requirements and have operational characteristics and failure modes that are well understood. NASA has the responsibility to participate with the CEV contractor in the development of requirements for the relative navigation sensors, and also to perform an independent assessment of the ability of the sensors to meet requirements. In order to do this, NASA will need to perform independent testing as early as possible in order to minimize cost and schedule impacts.

The first year of this project (Fiscal Year 2006) was focused on a series of "pathfinder" tasks to develop test plans, test facility requirements, trajectories, math model architecture, simulation platform, and processes. Four highly applicable candidate sensors were used as test articles for the "pathfinder" activities: the Johnson Space Center's (JSCs) Automatic Targeting and Reflective Alignment Concept (AutoTRAC) Computer Vision System (ACVS), which is a camera-based system that uses reflectors on the target vehicle; JSCs Natural Feature Image Recognition (NFIR), which is a camera-based system that does not require reflectors; Marshall Space Flight Center's (MSFCs) Advanced Video Guidance Sensor (AVGS), which is a laser-based system that uses reflectors on the target vehicle; and the Optech Light Detection and Ranging (LIDAR), which is a laser-based system that produces range and intensity data, provided by the Jet Propulsion Laboratory (JPL) for this task. Sensor characterization testing for each of these four sensors was conducted at the MSFC Flight Robotics Laboratory (FRL) using the FRL 6-Degree-of-Freedom (DOF) gantry system, called the Dynamic Overhead Target Simulator (DOTS). 
The target vehicle for "docking" in the laboratory was a mockup that was representative of the proposed CEV docking systems, with added retroreflectors for the pathfinder sensors and a standoff cross target for visual recognition by the NFIR sensor.

The second year of this project (Fiscal Year 2007) is focused on follow-on testing activities, including dual-AVGS testing at the FRL, and integration and testing for two of the pathfinder sensors at the Johnson Space Center's Six-DOF Dynamic Test System (SDTS). The SDTS will be the primary testing location for the Orion spacecraft's Low Impact Docking System (LIDS). Project team members have integrated the Orion 6-DOF simulation, called Architecture for Exploration Studies (ANTARES), with the SDTS computer system so that real-time closed loop testing can be performed with relative navigation sensors and the docking system in the loop during docking and undocking scenarios.

\section{SDTS}

The general purpose of the SDTS, shown in Figure 1, is to demonstrate and verify on-orbit tasks such as assembly of space structures using berthing and docking. The SDTS is a closed loop dynamic testing system that combines high fidelity test articles and software models to produce an integrated simulation of two-body contact in free space. Common uses of the SDTS include engineering evaluation of mechanical device operation, verification of in-space assembly tasks, development of crew training and operational procedures, and demonstration of advanced robotics technologies

SDTS provides a six degree-of-freedom motion base platform, a superstructure for mounting test articles and test sensors, an intercom network for voice communications, and a High Speed Data Acquisition System (HSDAS). In addition, it provides multiple control bay workstations as well as three different dynamic and kinematic simulations to control the motion platform; a two-free-body docking simulation, a Space Shuttle Remote Manipulator System (SRMS) berthing simulation, and a Space Station Remote Manipulator System (SSRMS) berthing simulation which includes Orbital Boom Sensor System (OBSS) dynamics.

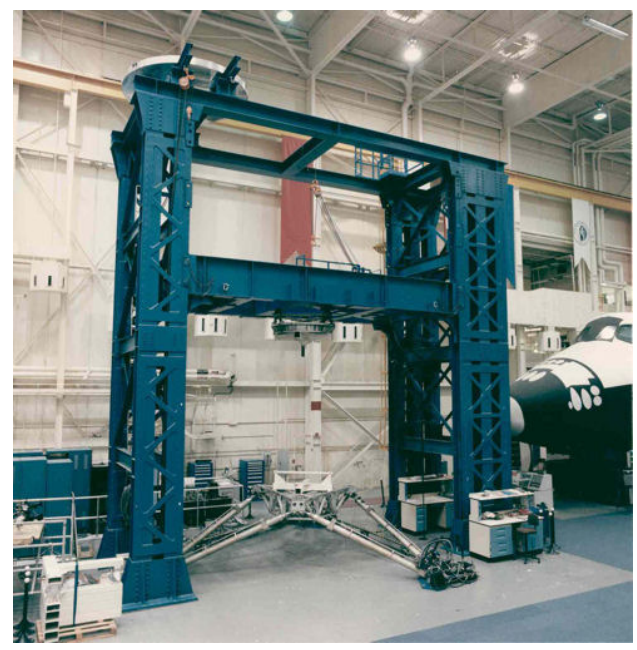

FIGURE 1. SDTS Facility.

The SDTS range of motion is shown in Figure 2, and the specifications are in Table 1. 


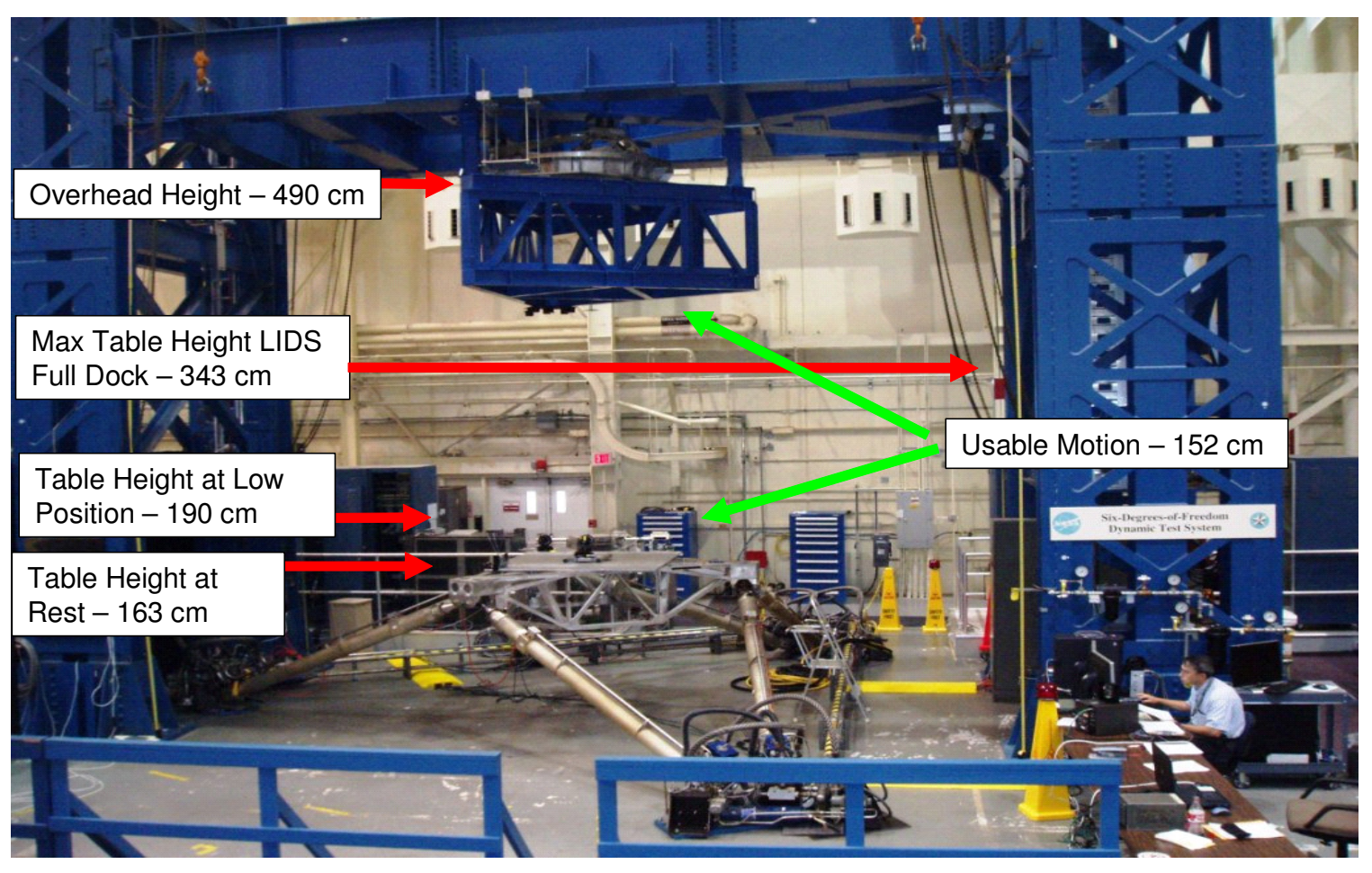

Figure 2. SDTS Range of Motion.

TABLE 1. SDTS Specifications. [Jenny needs to convert units to SI]

\begin{tabular}{|c|c|}
\hline \multicolumn{2}{|l|}{ Pressure } \\
\hline Operating Pressure: & 3000 (high) or 1000 (low) psi \\
\hline \multicolumn{2}{|l|}{ Load Capacity } \\
\hline Platform Maximum Force: & $10,000 \mathrm{lbf}$ \\
\hline \multicolumn{2}{|l|}{ Data Acquisition } \\
\hline Total number of data acquisition channels available: & 96 analog \\
\hline \multicolumn{2}{|l|}{ Performance } \\
\hline Platform clearance range to overhead attachment surface: & 30-282 inches \\
\hline $\begin{array}{l}\text { Maximum test article clearance width, lateral (Y), (distance between } \\
\text { superstructure uprights): }\end{array}$ & 336 inches \\
\hline Platform movement maximum extent, vertical (X): & $+/-124$ inches \\
\hline Platform movement maximum extent, lateral $(\mathrm{Y})$ : & $+/-40$ average +70 peak inches \\
\hline Platform movement maximum extent, lateral $(\mathrm{Z})$ : & $+/-40$ average, $+/-62$ peak inches \\
\hline Platform movement maximum extent, angular: & $+/-20$ degrees \\
\hline Leg actuator velocity, short duration maximum: & 20 inches per second \\
\hline Table Bandwidth: & $6-9 \mathrm{~Hz}$ \\
\hline Incremental Accuracy: & 0.006 inch \\
\hline Absolute Accuracy: & $\sim 0.1$ inch \\
\hline
\end{tabular}

Truth data for the SDTS trajectories are obtained with the use of an Optotrak laser system. The Optotrak [will provide specs here on how good it is]

\section{ORION SIMULATION}

[To be provided in final version] 


\section{TEST ARTICLES}

Two test articles were selected for testing at the SDTS: ACVS and NFIR. The ACVS technology uses the "spot pose" technique that is common with AVGS and other candidate relative navigation systems, in which a sensor uses a cluster of targets in a known pattern to extract six-DOF state, or "pose" as the geometric result. NFIR is designed to use one or more cameras and uses image-matching of target vehicle features with known locations. These two sensors were primarily selected because domain expertise for both sensors is resident at JSC and they could serve as inexpensive, but applicable, pathfinder sensors for the SDTS integration activities. Additional details about the test articles are provided in this section.

\section{AutoTRAC Computer Vision System (ACVS)}

The ACVS is a camera-based system that employs the use of light emitting diodes (LEDs) and specific targets composed of either mirrors or reflective surfaces to determine a relative state (range, azimuth, elevation, and roll, pitch, and yaw). ACVS elements are shown in Figure 3.



FIGURE 3. AutoTRAC Computer Vision System (ACVS)

The ACVS is composed of a charge-coupled device camera with LED array using a specific target. The operating principle is a 6-DOF pose based on a known target pattern. The first video frame is taken with LEDs on; the second video frame with LEDs off. The two frames are then subtracted from each other, eliminating the background while leaving the bright return from the retroreflectors. Standard image segmentation techniques are then used to determine the retroreflectors' locations in the image. The correspondence between the potential target features (retroreflector blobs) and the known target model is then determined. Finally, a non-linear least-squares fit of the image data is done to determine the 6-DOF pose of the target object with respect to the camera. Each ACVS pose is independent of the previous pose, with no external "positioning" inputs needed. Multiple ACVS targets are supported which would allow for pose results at long range using a large target (LRT), while using a smaller target (SRT) at close range

The ACVS was used for the Dexterous End Effector Detailed Test Objective (DTO) for STS-62, DTOs for STS-85 and STS95, and the Miniature Autonomous Extravehicular Robotic Camera (Mini AERCam) navigation sensor for docking. The ACVS hardware and software components used for this test program are based on a mid 1990's development effort at JSC with occasional enhancement efforts over the last several years. 
TABLE 2. ACVS FRL Operational and Performance Parameters

$\begin{array}{ll}\text { Field of View } & \text { 28 degrees horizontal x } 21 \text { degrees vertical - one fixed 9mm lens } \\ \text { Range } & 0.75-40 \text { meters. SRT: approximately } 0.75-20 \text { meters LRT: approximately } 8-40 \text { meters. } \\ \text { Accuracy } & \text { Predicted accuracies of } 0.001 \text { inch and } 0.1 \text { degree } \\ & \text { Operational expectation is } 1 \text { percent of range and } 1 \text { degree in attitude. } \\ \text { Update rate } & 15 \mathrm{~Hz} @ 30 \mathrm{hz} \text { frame rate } \\ \text { Target angles } & \text { LRT/SRT up to } \pm 10 \text { degrees } \\ \text { Power } & 7.2 \text { watts @ } 18 \text { Volts DC } \\ \text { Mass } & 0.45 \text { kilograms each }- \text { sensor/LED and electronics box } \\ \text { Dimensions } & 12 \times 10 \times 10 \text { centimeters sensor, } 13 \times 13 \times 6 \text { centimeters electronics } \\ \text { Data interface } & \text { IEEE } 1394 \text { from the ACVS camera to the data processing computer }\end{array}$

\section{Natural Feature Image Recognition (NFIR) Overview}

NFIR is also a camera-based system, as shown in Figure 4. Instead of using a specific target illuminated by LEDs as ACVS does, NFIR can use any high contrast features on a target of interest for computing motion and pose, as long as a 3D geometric model of the target exists, and a rough estimate of initial pose is available, either from auto/manual acquisition or from other sources. 2D motion of images features are used to compute 3D motion of the target that is in turn used to update the pose. A Kalman filter is used estimate state (position, orientation and first-time derivative) from the pose, and to predict locations of image features in the next image frame.

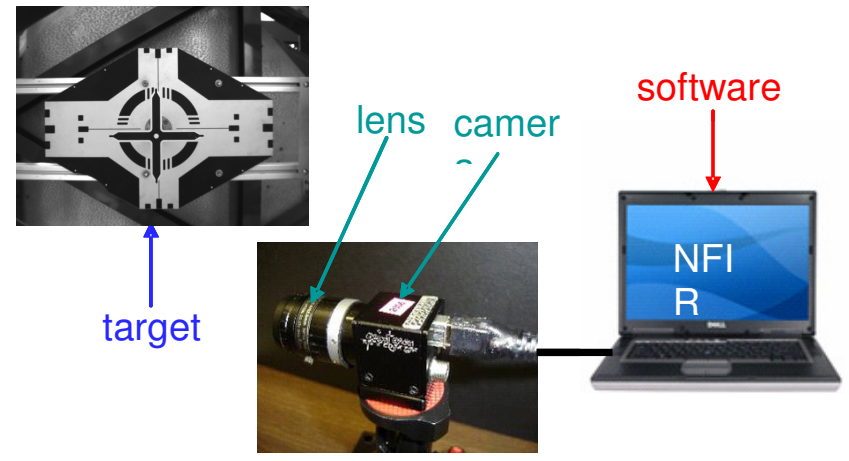

Figure 4: Natural Feature Image Recognition (NFIR)

NFIR was tested on more than eighty trajectories, ranging from 40 meters to 1 meter, at Marshall Space Flight Center. Point Gray Flea $2(640 \times 480)$ cameras with $25 \mathrm{~mm}, 16 \mathrm{~mm}$, or $9 \mathrm{~mm}$ focal length lens were used for these tests. The object of interest was a mockup of the ISS docking adapter, including its docking target with stand-off cross. In general, NFIR tracked the target fairly well, even with the solar simulator on, in low light conditions, when part of target was occluded (as long as there were enough features visible for pose computation). Accuracy of the test is summarized in Table 3. Accuracy was gradually degraded when the target was far away and its size in the image was decreasing, was over-exposed, or was only faintly illuminated.

TABLE 3: NFIR Operational and Performance Parameters.

$\begin{array}{ll}\text { Range accuracy } & \pm 1 \text { percent of range } \\ \text { Attitude accuracy } & \pm 1 \text { degrees } \\ \text { Data output rate } & 5-10 \mathrm{~Hz}\end{array}$

NFIR was also tested using simulated video of the H-II Transfer Vehicle (HTV) as seen from an exterior ISS camera from a range of 260 meters to 10 meters, and using simulated video of the Hubble Space Telescope aft bulkhead during terminal approach. Comparable accuracy was obtained from both tests. 


\section{DOCKING TARGETS FOR THE CLOSED LOOP TEST}

Because this test occurs at close range (from ten feet to contact), the NFIR uses only the docking target with standoff cross rather than the docking adapter mock-up that was used in the FRL test. The docking target, shown in Figure 5, is nearly identical to the one that is used by Shuttle when it docks to the International Space Station (ISS). In addition, because of the short range only one camera with a fixed focal length lens is used. The ACVS uses a target with a set of five retro-reflectors (one out-of-plane) with a mirror in the center, shown in Figure 6.



FIGURE 5. ACVS and NFIR Targets Mounted to SDTS.

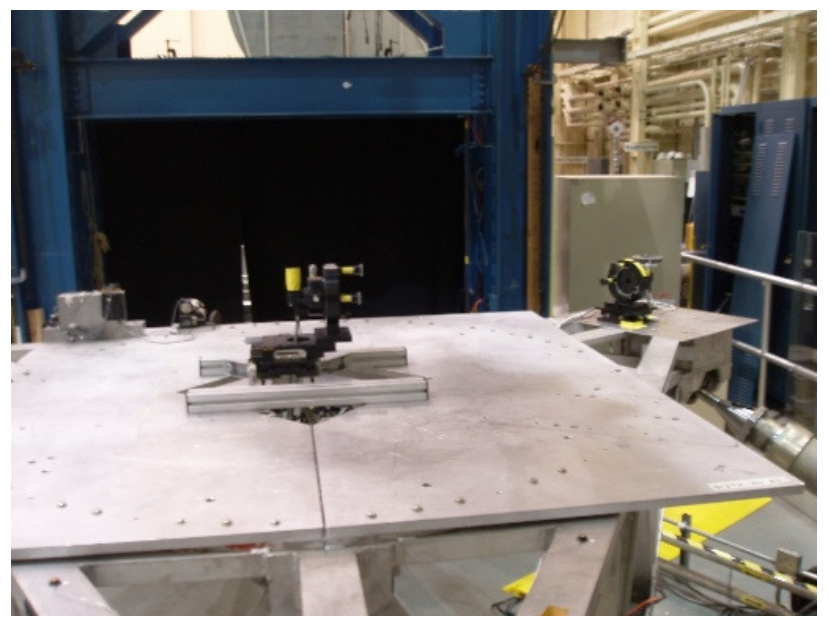

FIGURE 6. NFIR and ACVS Sensors

\section{TEST TRAJECTORIES}

The test sensors undergo three initial calibration test runs each morning. The calibration trajectories are intended to ensure that the sensors are performing consistently from day-to-day. The three tests are:

1) The Radial Trajectory. In the radial test, the test stand is maneuvered from 3 feet to 0 feet along the boresite of the docking cross. There is no lateral motion of the test stand and there is no relative rotation of the test stand with respect to fixed stand. The test objective is to ensure that the bore site of the sensor is in alignment.

2) The Diamond Trajectory. The diamond test applies lateral motion to the test stand at a 1.5 foot stand-off distance. The lateral motion is a +/- 1 foot off-axis and forms a diamond in the X-Y axes. This trajectory tests the sensors offaxis capabilities.

3) The Decaying Error Trajectory (DET). The DET test varies all translational and rotational components of the test stand relative to the fixed stand. The lateral variations have a maximum of 3 feet and vary sinusoidally to 0 at docking. Likewise, the relative roll, pitch and yaw offsets are maximum of 5, 2, and 3 degrees at the start of the trajectory and vary sinusoidally to 0 at docking. This trajectory is designed to provide distinctive signatures in all axes for sensor calibration and testing.

Following the initial calibration runs, three nominal Crew Exploration Vehicle (CEV) docking trajectories are executed. The docking trajectories are controlled by a Trick simulation environment that is designed to demonstrate the approach of the CEV to the International Space Station (ISS). The three test trajectories are:

1) A nominal approach trajectory, which has the initial conditions of the CEV docking port located 3 feet from the ISS docking adapter,

2) A positional offset approach trajectory, which has the initial conditions of the CEV 0.5 foot offset in both lateral axes,

3) And a yaw offset approach trajectory, which has the initial conditions of the CEV located 3 feet from the ISS at an initial yaw angle of 5 degrees. 
Each of the tests utilizes accurate mass properties of the CEV and prototype Guidance \& Control algorithms. Initially, the navigation is accomplished via simulated perfect sensors. Later testing replaces the idealized sensors and uses the real sensors under test as inputs into the navigation algorithm; thereby testing the sensor in a closed loop manner around the full GN\&C simulated system.

\section{TEST RESULTS}

To be provided after testing is complete in August 2007

\section{CONCLUSIONS}

This key result of this activity is the development a comprehensive six degree-of-freedom laboratory test program that will be applied during testing of CEV and other spacecraft sensors. Test plans, procedures, scripts, processes, and trajectories can be used for future tests. [Jenny to update after conclusion of tests]

\section{ACKNOWLEDGEMENTS}

The authors would like to acknowledge contributions to this paper from other team members, including [will list other SDTS and project support personnel].

\section{REFERENCES}

To be added. 